ARTICLE

DOI: $10.1038 / s 41467-017-00893-x$

\title{
Color-selective photodetection from intermediate colloidal quantum dots buried in amorphous-oxide semiconductors
}

Kyung-Sang Cho ${ }^{1,2}$, Keun Heo ${ }^{2,3}$, Chan-Wook Baik ${ }^{1,2}$, Jun Young Choi ${ }^{3}$, Heejeong Jeong ${ }^{1,5}$, Sungwoo Hwang ${ }^{1,2} \&$ Sang Yeol Lee ${ }^{4}$

We report color-selective photodetection from intermediate, monolayered, quantum dots buried in between amorphous-oxide semiconductors. The proposed active channel in phototransistors is a hybrid configuration of oxide-quantum dot-oxide layers, where the gatetunable electrical property of silicon-doped, indium-zinc-oxide layers is incorporated with the color-selective properties of quantum dots. A remarkably high detectivity $\left(8.1 \times 10^{13}\right.$ Jones $)$ is obtained, along with three major findings: fast charge separation in monolayered quantum dots; efficient charge transport through high-mobility oxide layers $\left(20 \mathrm{~cm}^{2} \mathrm{~V}^{-1} \mathrm{~s}^{-1}\right)$; and gatetunable drain-current modulation. Particularly, the fast charge separation rate of $3.3 \mathrm{~ns}^{-1}$ measured with time-resolved photoluminescence is attributed to the intermediate quantum dots buried in oxide layers. These results facilitate the realization of efficient color-selective detection exhibiting a photoconductive gain of $10^{7}$, obtained using a room-temperature deposition of oxide layers and a solution process of quantum dots. This work offers promising opportunities in emerging applications for color detection with sensitivity, transparency, and flexibility.

\footnotetext{
${ }^{1}$ Samsung Advanced Institute of Technology, Device Lab, Suwon 16678, South Korea. ${ }^{2}$ Samsung Advanced Institute of Technology, Research Center for Time-Domain Nano-Functional Device, Suwon 16678, South Korea. ${ }^{3}$ School of Electrical Engineering, Korea University, Seoul 02841, South Korea.

${ }^{4}$ Department of Semiconductor Engineering, Cheongju University, Cheongju 28503, South Korea. ${ }^{5}$ Present address: Department of Physics and Institute for Advanced Study, The Hong Kong University of Science and Technology, Clear Water Bay, Hong Kong, China. Kyung-Sang Cho, Keun Heo, and Chan-Wook Baik contributed equally to this work. Correspondence and requests for materials should be addressed to K.-S.C. (email: k-s.cho@samsung.com) or to S.H. (email: swnano.hwang@samsung.com) or to S.Y.L. (email: sylee@cju.ac.kr)
} 
T he detection of light has historically been one of the most fundamental and academic subjects behind various optoelectric applications ${ }^{1-11}$. At present, the development of photodetectors, which convert incident photons to electrical signals, has confronted significant challenges regarding the realization of efficient and sensitive detection with low noise for the ultraviolet $(\mathrm{UV})^{3,4}$, visible $^{5-9}$, and infrared regimes ${ }^{10}, 11$ of electromagnetic spectrum. Furthermore, a strong demand for complementary metal-oxide semiconductor (CMOS)-compatible, monolithic integration with a low-cost and simple fabrication process has arisen, which may not be achievable using epitaxially grown III-V semiconductors for photoconductors, avalanche photodiodes, and photomultipliers. Such devices have disadvantages related to material growth conditions, high-voltage operation, and bulkiness, including increased fabrication cost and complexity. However, colloidal quantum dots (QDs), often referred to as semiconductor nanocrystals, are easily processible for integration onto various substrates using a low-cost, solutioncoating method. They have unique optical properties, such as bandgap energies tunable by adjusting their sizes, narrow emission bandwidths, broad absorption spectrum, and high photoluminescence quantum efficiencies. These outstanding advantages have incited considerable research efforts toward the development of QD photodetectors ${ }^{1-11}$, which now target the performance of conventional state-of-the-art photodetectors.

One of the figures of merit characterizing photodetection is the normalized detectivity $\left(D^{*}\right)$, expressed in units of Jones $\left(\mathrm{cm} \mathrm{Hz}^{1 / 2} \mathrm{~W}^{-1}\right)$, equal to the square root of the optically active area divided by its noise equivalent power (NEP). Konstantatos et al. ${ }^{5}$ have demonstrated visible and near-infrared (NIR) $)^{10}$ photoconductive detectors using PbS colloidal QDs, and reported $D^{*}$ values of $5 \times 10^{12}$ and $1.8 \times 10^{13}$ Jones, respectively. Their achievement in the visible spectrum is comparable to the results for conventional silicon photodiodes $\left(\sim 4 \times 10^{12} \text { Jones }\right)^{2}$, because of the relatively high mobility $\left(10^{-2} \sim 1 \mathrm{~cm}^{2} \mathrm{~V}^{-1} \mathrm{~s}^{-1}\right)$ of the functionalized PbS QDs caused by the large wavefunction overlap. Lee et al. ${ }^{6}$ have reported visible photoconductors consisting of CdSe colloidal QDs capped with an $\mathrm{In}_{2} \mathrm{Se}_{3}$ metal chalcogenide complex, and achieved a $D^{\star}$ of $1 \times 10^{13}$ Jones, contributed by the high-mobility $\left(16 \mathrm{~cm}^{2} \mathrm{~V}^{-1} \mathrm{~s}^{-1}\right)$. The purpose of that study was to obtain high mobility via ligand exchange, due to the intrinsically low mobility of QDs $\left(10^{-6} \mathrm{~cm}^{2} \mathrm{~V}^{-1} \mathrm{~s}^{-1}\right)$ for visible detection ${ }^{12,13}$.

In contrast to photoconductor devices, phototransistors provide a wider degree of photocurrent control, which is achievable by adjusting the gate voltages $\left(V_{\mathrm{G}}\right)$ as well as the source-drain voltages and incident light intensities. Recently, QD-hybrid phototransistors have been introduced for use in combination with high-mobility materials, e.g., amorphous-oxide semiconductors (AOSs) $^{7-9}, 14$, graphene ${ }^{11}$, and 2-D materials ${ }^{15}, 16$. $\mathrm{PbS}$ colloidal QDs on mechanically exfoliated graphene flakes, for example, have exhibited device $D^{*}$ values as high as $7 \times 10^{13}$ Jones, with a responsivity $(R)$ of $\left.10^{7} \mathrm{AW}^{-111}\right)$, the ratio of the generated photocurrent to the incident optical power. The high electron mobility (c.a. $1000 \mathrm{~cm}^{2} \mathrm{~V}^{-1} \mathrm{~s}^{-1}$ ) of graphene contributes to the fast hole transfer, while the electrons remain in trap states. However, interface control between QDs and graphene remains an issue 2,17 .

On the other hand, AOS materials with wide bandgap energies have attracted particular attention in the fields of electronics and optoelectronics ${ }^{4}, 18-23$. The advantages of AOS films are their high transparency, low processing temperature, smooth surface with no grain boundary, and high electron mobility regardless of the degree of film disorder ${ }^{8-21}$. The application of the majority of AOS materials to visible wavelength region remains challenging, because of their intrinsically wide bandgaps. That is, the use of AOSs for photodetection is, in principle, limited to
UV energy band ${ }^{4,22}$. However, continuous attempts have been made to use AOSs in image sensors or phototransistors, in parallel with the development of active-matrix thin-film transistors (TFTs) for use in display devices ${ }^{18}, 23$, to replace amorphous or poly silicon. Recently developed AOS films as channel materials in TFTs demonstrated high electron mobilities of more than $30 \mathrm{~cm}^{2} \mathrm{~V}^{-1} \mathrm{~s}^{-118}$ ). For example, In-Ga-Zn-O (IGZO) and Ge-doped In-Ga-O (Ge-IGO) layers have been used as highmobility channels in conjunction with CdSe QDs located on either the top or bottom surfaces of the oxide layers ${ }^{7-9}$. In the channel configuration of QDs on oxide (QO), i.e., QDs on IGZO, $R$ reached an order $10^{4} \mathrm{AW}^{-1}$ in UV range, while a photocurrent saturation was observed for an input light intensity of more than $300 \mu \mathrm{W} \mathrm{cm}{ }^{-27}$ ). In this case, the charge separation from QDs to AOS is considered to be dependent on the degree of contact between QD and-AOS. In addition, phototransistors with $\mathrm{PbS}$ QDs on IGZO demonstrated a photoresponsivity of $10^{6} \mathrm{AW}^{-1}$ in NIR range ${ }^{14}$. Thus, the demonstration of photodetection using QDs with AOS offers promising opportunities, including potentially promising multispectral photodetection in visible spectrum, but has yet to yield high $R$ and $D^{*}$.

Here, we propose a hybrid QD-AOS phototransistor composed of an oxide-QD-oxide (OQO) layered channel, in which the intermediate QDs are buried in between the top and bottom oxide layers. This may provide a fast charge separation from QD to AOS layer by the increase of interface area between QD and AOS. Amorphous SIZO (Si-doped In-Zn-O) films are chosen to allow room-temperature, radio-frequency (RF) sputtering and low-temperature, annealing processes ${ }^{20}, 21$. We employ CdSe, CdSeS, and CdS QDs for red (R), green (G), and blue (B) color detection, respectively, using their particular absorption characteristics. As an example of color-selective detection, we demonstrate a logic circuit comprised of an electro-optical NOT gate using different wavelength illuminations. This hybrid OQO configuration is expected to achieve highly sensitive photodetection because of fast charge separation induced by the large interfacial areas of QDs buried in the oxide layers, along with the fast carrier transport caused by the high mobility of the oxide layers. In particular, a rigorous characterization of $D^{*}$ with respect to the noise spectrum is conducted as a function of modulation frequency for the proposed hybrid OQO phototransistors.

\section{Results}

Photoresponse of hybrid QD-AOS phototransistors. A schematic of the proposed hybrid OQO phototransistor is shown in Fig. 1. The OQO layer is located between the source and drain electrodes, to absorb the incident light, generate photocarriers, and to transport the carriers to the electrodes under the control of the back-gated voltages. The transmission electron microscopy (TEM) image (Fig. la, inset) shows the spin-coated QD layer buried in between the top and bottom SIZO layers. We chose SIZO as a channel material because it exhibits high mobility in the amorphous phase via controlled doping of silicon in the IZO. An electron mobility greater than $20 \mathrm{~cm}^{2} \mathrm{~V}^{-1} \mathrm{~s}^{-1}$ is provided by the room-temperature RF-sputtered SIZO layers, following a $150{ }^{\circ} \mathrm{C}$ annealing process ${ }^{20}$. In contrast, an annealing temperature of more than $300^{\circ} \mathrm{C}$ is typically required for other oxide materials, e.g., In-Ga-Zn-O, Zn-Sn-O, and In-Ga- ${ }^{18}$. We measured the mobilities of the OQO films for R, G, and B QDs buried in the SIZO layers and for different QD layer thicknesses, along with that of a 40-nm SIZO layer without QDs (see Supplementary Table 1). Hence, we found that the Ds buried in the SIZO layers might not affect the SIZO channel mobility. Figure $1 \mathrm{~b}$ shows the photocurrent mapping of the OQO phototransistor, indicating that the photocarrier generation occurs across the entire OQO area, not from the electrodes or their edges. Figure $1 c$, d show the 


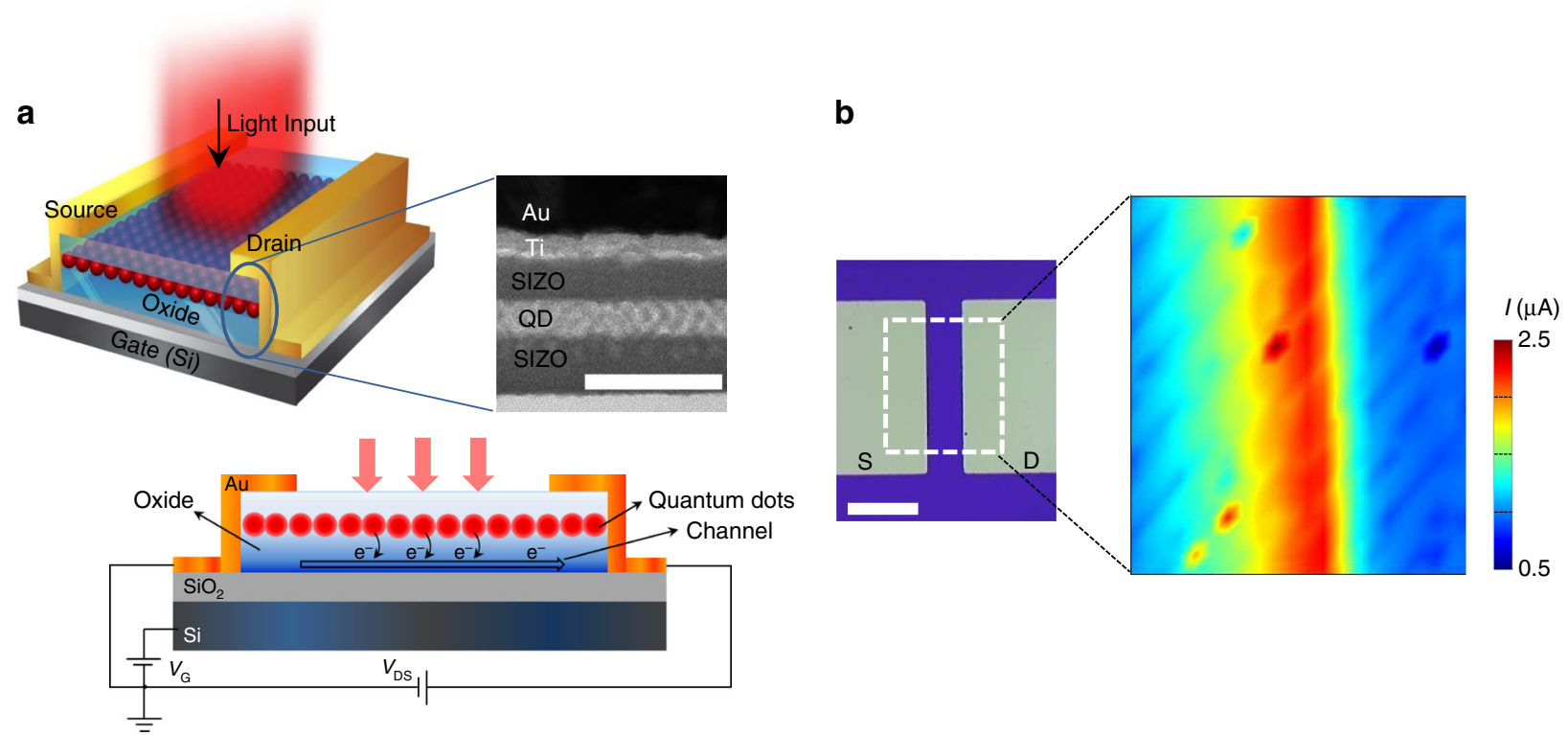

C

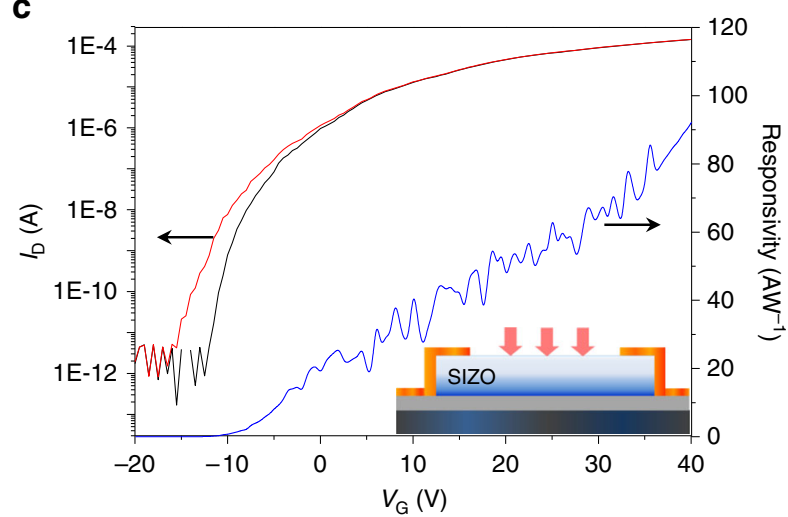

d

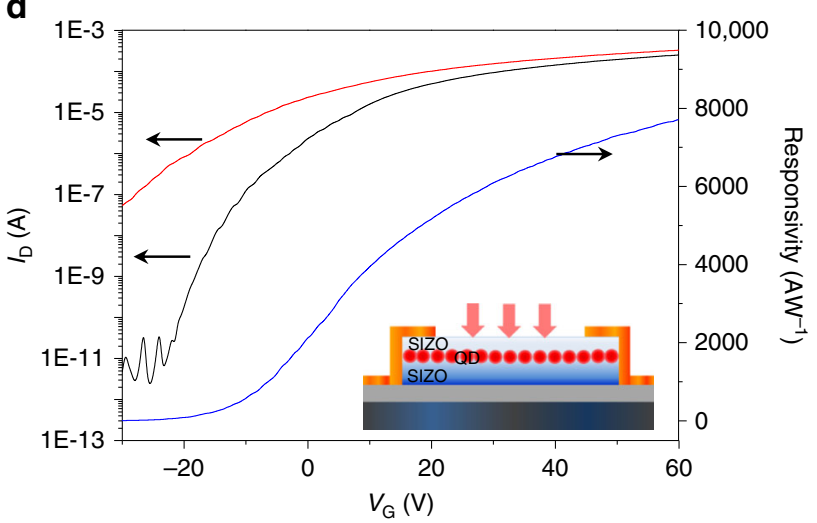

Fig. 1 Hybrid phototransistors of quantum dots buried in amorphous-oxide semiconductors. a Schematics of proposed device structure. The channel contains OQO layers, with QDs buried between the top and bottom oxide (SIZO) layers, as shown in the cross-sectional TEM image. Scale bar is 100 nm. The top and bottom SIZO layer thicknesses are 30 and $40 \mathrm{~nm}$, respectively. The 1-6 QD layers have 5.5-30-nm thicknesses. The photocarriers generated by the QDs via light absorption are transported through the oxide layers. b Spatial photocurrent profile for 487-nm laser beam with 10-nW power. Scale bar is $100 \mathrm{~nm}$. The photocurrent was measured during laser beam scanning across the photodetector surface. c, d Photocurrent response and $R$ of (c) SIZOonly device and (d) monolayered-red-QD (1RQ) OQO device, at 487-nm illumination wavelength with 10-nW power. $V_{S D}$ is $5 \mathrm{~V}$. Black and red lines are dark current and photocurrent, respectively. Responsivities are indicated by blue lines

transfer characteristics, together with the $R$ behaviors of a singlelayered SIZO transistor and a monolayered-red-QD OQO (1RQOQO) transistor, respectively, under dark and light irradiation. In the SIZO-single-layered device, there is almost no absorption of input light at 487-nm wavelength because of the wide bandgap energy of $3.2 \mathrm{eV}^{24}$ ) (see Supplementary Fig. 2). On the contrary, the measured photocurrent of the OQO device increases dramatically via photocarrier generation owing to the QD light absorption, as depicted in Fig. 1d. The $R$ of the SIZO-singlelayered device without QDs is less than $100 \mathrm{AW}^{-1}$; however, this value increases to more than $6000 \mathrm{AW}^{-1}$ in the 1RQ-OQO device, where $R=\left(I_{\mathrm{SD}}\right.$, light $-I_{\mathrm{SD}}$, dark $) / P_{\text {light }}\left(\mathrm{AW}^{-1}\right)$, where $I_{\mathrm{SD}}$, light is the drain current under irradiation of light, $I_{\mathrm{SD}}$, dark is the drain current under dark, and $P_{\text {light }}$ is the irradiation light power ${ }^{2}$ (see also Supplementary Fig. 3 as depicted in linear scale).

Photocarrier generation and transport. The energy-band diagram and the operating principles of the OQO phototransistors are shown in Fig. 2a, b, respectively. The bandgap and band positions of the QD and SIZO are determined from the measurement of ultraviolet/visible (UV/Vis) absorption spectrum, UV photoelectron spectroscopy, electron energy loss spectrum, and Kelvin probe method ${ }^{21}$. The QD Fermi level, 4.4 $\mathrm{eV}$, is slightly higher than that of SIZO, $4.2 \mathrm{eV}$, which allows charge separation via QD light absorption. The photocarriers generated by the charge separation flow with respect to the potential difference $\left(V_{\mathrm{D}}\right)$ between the source and drain electrodes under the control of $V_{\mathrm{G}}$, as illustrated in Fig. 2b. Figure $2 \mathrm{c}$ shows the color spectral $R$ trends of phototransistors with 4-layered-redQD OQO (4RQ-OQO), 6-layered-green-QD OQO (6GQ-OQO), and 6-layered-blue-QD OQO (6BQ-OQO) configurations (see also, Supplementary Fig. 4). The photocurrent response for each color follows the QD absorption, as shown in Fig. 2d. This implies that the photoresponse of the OQO phototransistors comes from the RGB photoexcitation of the QDs, rather than the defect-levelmediated carrier excitation of the SIZO. Because the SIZO bandgap energy $(3.26 \mathrm{eV})$ is larger than that of the QDs for visible spectrum, various kinds of QDs are applicable to the proposed OQO structure. Although QDs mostly have broad absorption spectrum, their color-dependent photoresponse provides spectral 
a
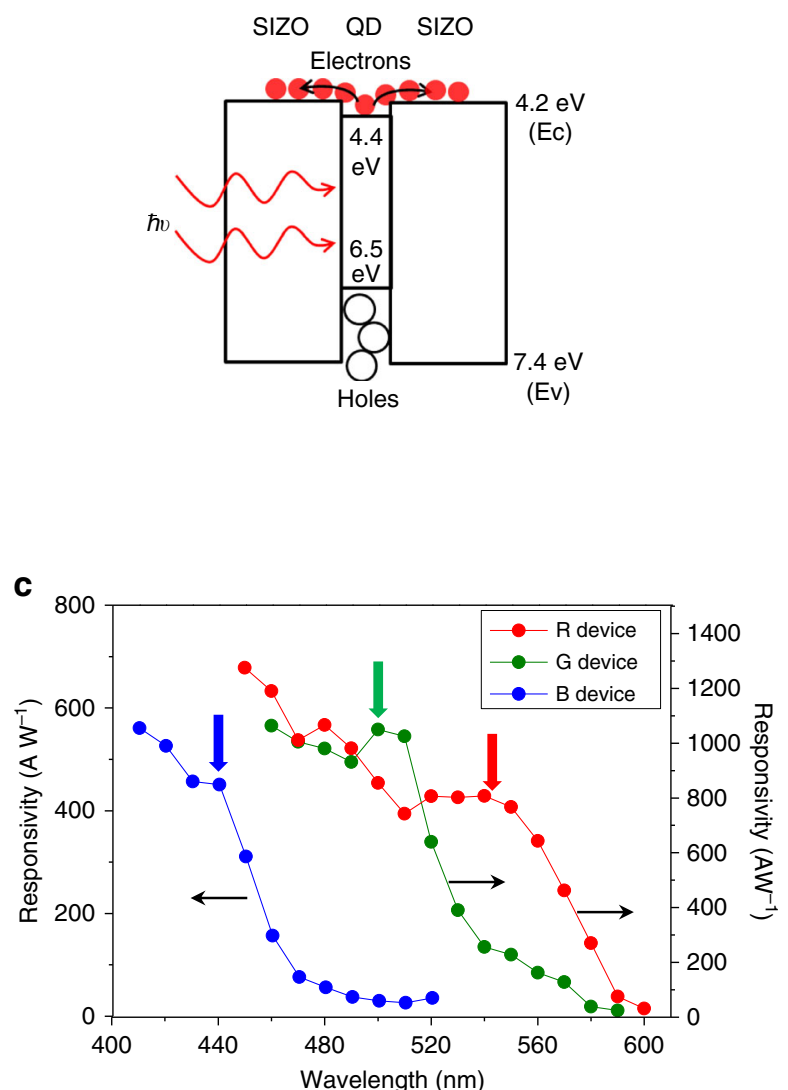

b
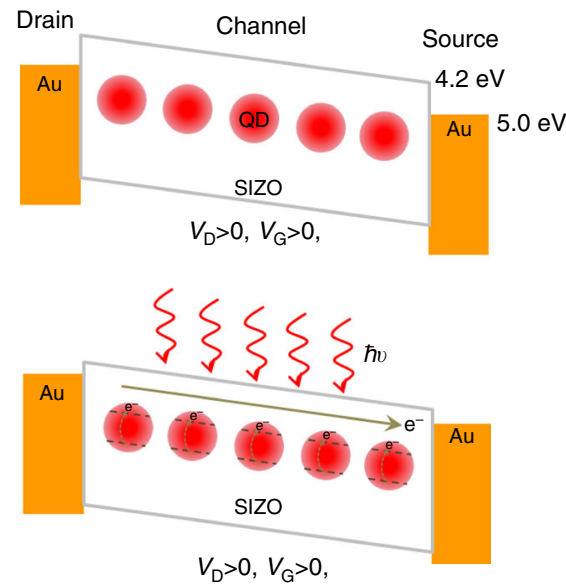

d

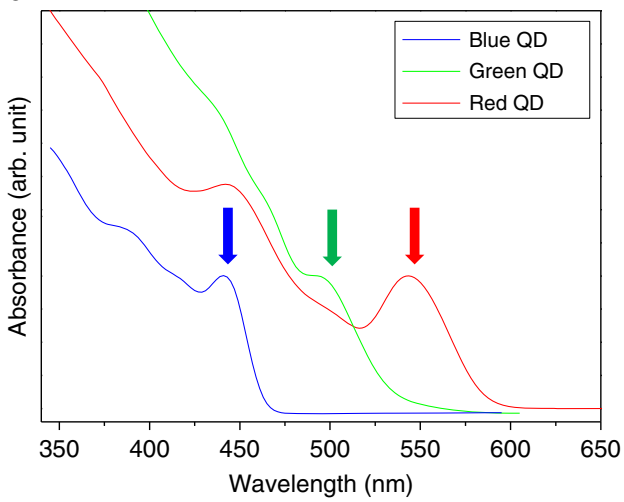

Fig. 2 Physical principles and spectral responses. a Energy diagram of red-QD OQO structure. $\mathbf{b}$ Light-induced carrier generation and transport mechanisms. c Spectral responses of $4 \mathrm{RQ}, 6 \mathrm{GQ}$, and 6BQ OQO phototransistors. d Absorption spectra of red, green, and blue QDs
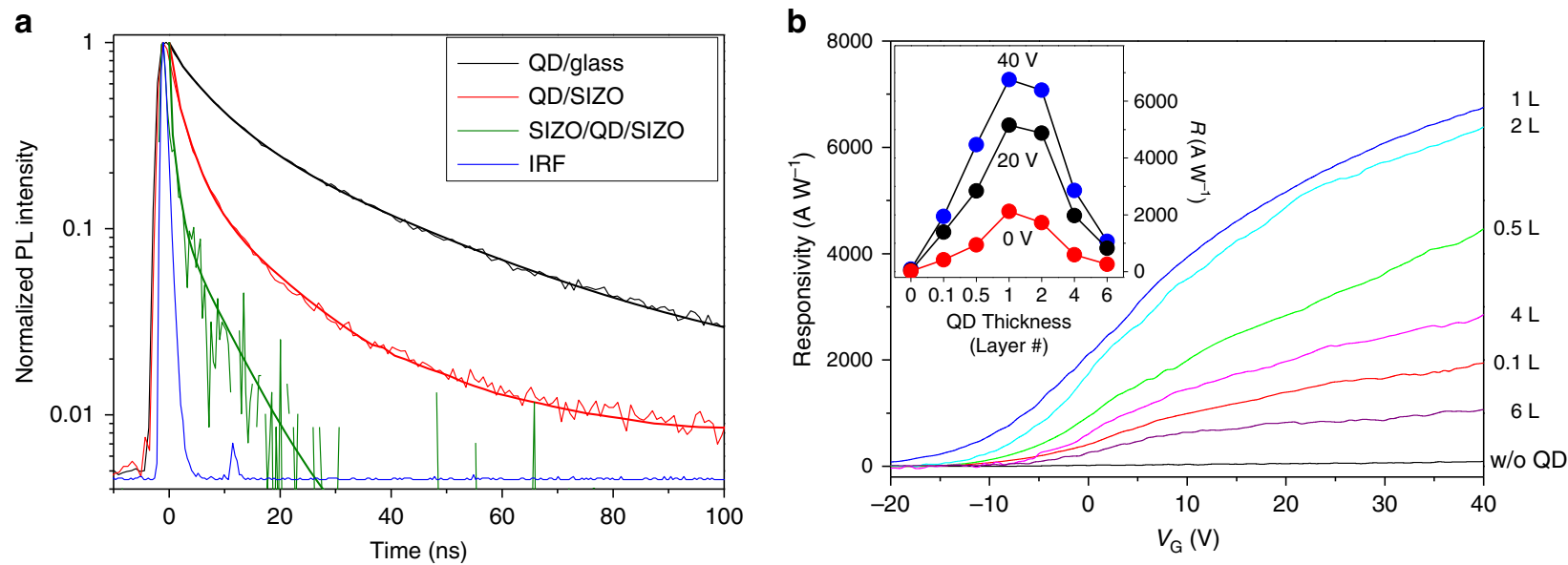

Fig. 3 Measurement results of fluorescence decays and photoresponses. a Fluorescence decay curves of three different monolayered-red-QD films, i.e., QDs, QDs on SIZO, and SIZO/QD/SIZO (OQO), all on glass substrates. The blue line is the system instrumental response function (IRF). The thicker solid lines are the fitting of each decay curves. $\mathbf{b} R$ vs. $V_{\mathrm{G}}$ with respect to QD layer thickness for red-QD OQO phototransistors. The incident light at $487-\mathrm{nm}$ wavelength is illuminated with $10-\mathrm{nW}$ power. In all, 0.1 and $0.5 \mathrm{~L}$ correspond to areal coverage of $10 \%$ and $50 \%$ of the monolayered-QD films, respectively. The inset figure shows the $R$ values vs. the QD layer thickness

information as well by appropriate signal processing method $^{25}$. Therefore, our OQO phototransistors can be usefully implemented in color-selective photodetection.

Characteristics in monolayered-QD OQO configuration. Figure 3a shows the fluorescence decay curves of three different monolayered-red-QD films (QD on glass, QD on SIZO, and
SIZO/QD/SIZO, i.e., OQO). The fluorescence decay time of the QD on SIZO is shorter than that of the QD on glass, because of the fast charge separation from the QDs to the SIZO film. The charge separation rate is calculated to be approximately $0.5 \mathrm{~ns}^{-1}$. Furthermore, the charge separation rate of the OQO film increases up to $3.3 \mathrm{~ns}^{-1}$, corresponding to more than six times that of the QD-on-SIZO film (see Supplementary Method 2). 

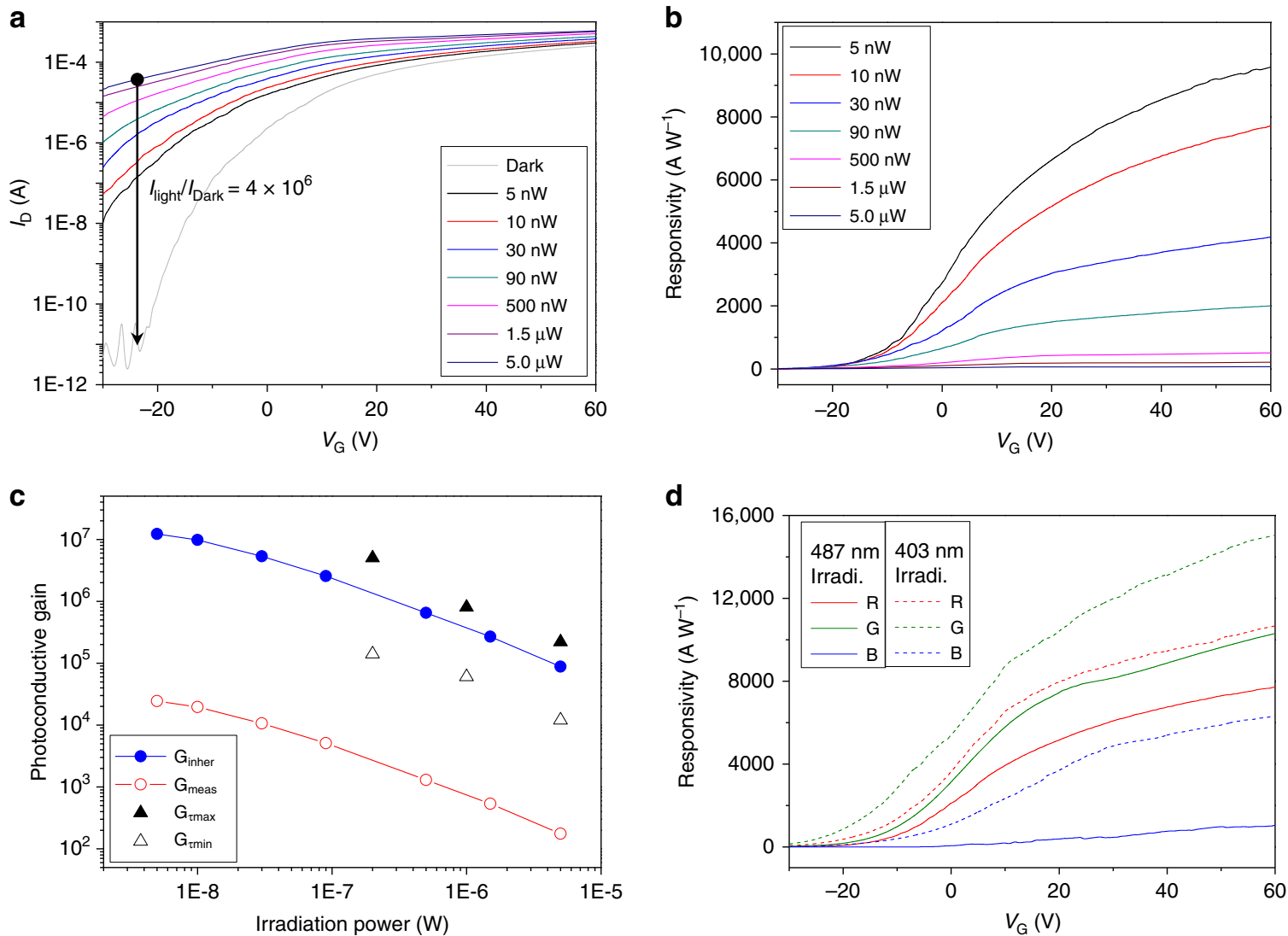

Fig. 4 Photoresponse characteristics and photoconductive gains. a Photocurrent responses and $\mathbf{b} R$ trends of $1 R Q-O Q O$ phototransistors with respect to incident light power. c Photoconductive gains using three different estimation methods vs. incident light power. d $R$ trends of 1RQ-, 1GQ-, and 1BQ-OQO phototransistors at 487- and 403-nm wavelengths with 10-nW power

This remarkable improvement stems from the increment of the effective interfacial area for the QDs buried in the amorphous SIZO films, i.e., the OQO structure, compared with the singleside contact experienced by the QDs on the SIZO film. The detailed discussion on the interfacial area is presented in Supplementary Fig. 5 and Supplementary Note 1. We further prepared and compared the photocurrent response of $\mathrm{QO}$ devices, i.e., monolayered-red-QD QO (1RQ-QO) phototransistors. They did not show a remarkable increase of $R$ compared to OQO configuration (see Supplementary Fig. 6).

We measured the $R$ of the proposed OQO structure vs. $V_{\mathrm{G}}$ with respect to the QD layer thickness, as depicted in Fig. 3b. The QD layer thickness varied from zero to six layers. In this figure, the " $0.1 \mathrm{~L}$ " and " $0.5 \mathrm{~L}$ " QD layers are sub-monolayered QD films, with areal coverages of $10 \%$ and $50 \%$ monolayered-QD films, respectively, (see Supplementary Figs. 7 and 8 for TEM and scanning electron microscope images for QD films). When the thickness increases up to one (mono) layer, i.e., the areal coverage becomes $100 \%$, the $R$ increases steadily at fixed $V_{\mathrm{G}}$; however, it begins to decrease once multiple layers are added. As illustrated in the inset of Fig. 3b, where $R$ is replotted against the QD layer thickness, the monolayered-QD OQO device exhibits the highest $R$. In our hybrid OQO phototransistors, the photoresponse mechanism can be described as follows: photocarrier generation via QD light absorption; charge separation from the QDs to the top and bottom SIZO layers; and carrier transport through the SIZO films to the electrodes. The photocurrent generation, which is proportional to the number of QDs, dominates up to monolayered-QD film structures; however, the charge separation rate and the carrier transport become important factors for 2-, 4-, and 6-layered QDs. In other words, the charge separation rate may not change, because the interfacial area between the QDs and their surrounding SIZO films does not effectively increase for structures with more than one-layer QD thickness. In particular, the photocarrier transport from QDs to the SIZO layers is thought to be disturbed significantly by the increasing number of charge trap sites in the multiple QD layer structures as discussed in the previous studies ${ }^{26-30}$. These have revealed low electronic conductivity in semiconductor nanocrystal arrays because of large concentrations of surface dangling bonds given by trap sites. More details are discussed in Supplementary Note 2. The above results show that the proposed hybrid OQO structure is superior to other configurations, when monolayered QDs are buried between the top and bottom amorphous SIZO layers.

Device performance of monolayered-QD OQO phototransistors. Figure $4 \mathrm{a}, \mathrm{b}$ show the transfer characteristics and $R$ behavior of 1RQ-OQO devices as functions of $P_{\text {light }}$ at $487-\mathrm{nm}$ wavelength. The on-off ratio $\left(I_{\text {light }} / I_{\text {dark }}\right)$ at $V_{\mathrm{G}}=-22 \mathrm{~V}$ is approximately $4 \times 10^{6}(66 \mathrm{~dB})$, as shown in Fig. $4 \mathrm{a}$. We successfully detected a wide range of incident light powers, corresponding to a range of three orders of magnitude $(5 \mathrm{nW}-5 \mu \mathrm{W})$. Note that the 1RQOQO configuration does not exhibit photocurrent saturation, even at $5 \mu \mathrm{W}$ (i.e., $255-\mathrm{mW} \mathrm{cm}^{-2}$ light intensity). This is one of the advantages of fast charge separation in OQO configuration. (see Supplementary Note 3). In contrast, a previous study of phototransistors with QDs on IGZO has reported saturation at a significantly lower light intensity of $300 \mu \mathrm{W} \mathrm{cm}{ }^{-2} 7$ ). In Fig. 4 b, $R$ is as high as $9800 \mathrm{AW}^{-1}$ at $60-\mathrm{V} V_{\mathrm{G}}$, for a 5 -nW incident light power (i.e., $255-\mu \mathrm{W} \mathrm{cm} \mathrm{cm}^{-2}$ light intensity). This result confirms the highly sensitive response of the OQO device. We also found the inverse relation between $R$ and the incident light power, which 
a

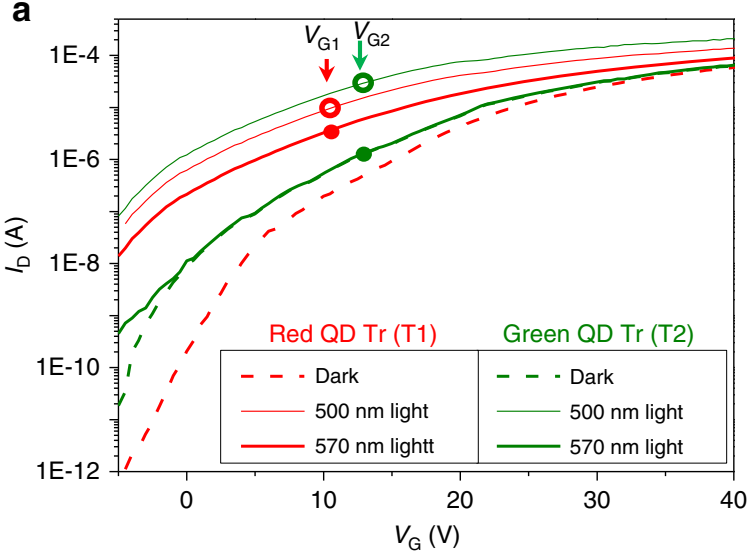

C

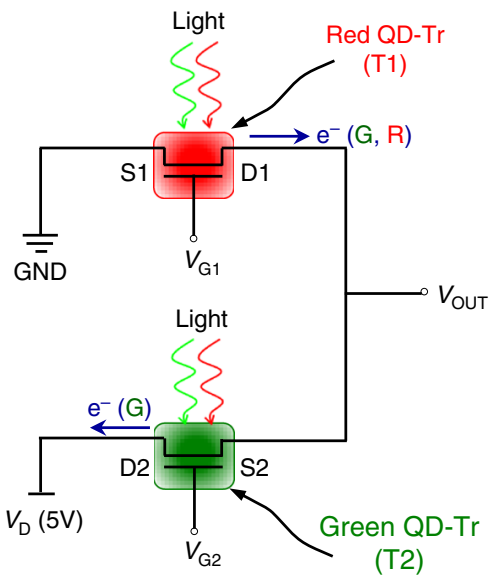

b

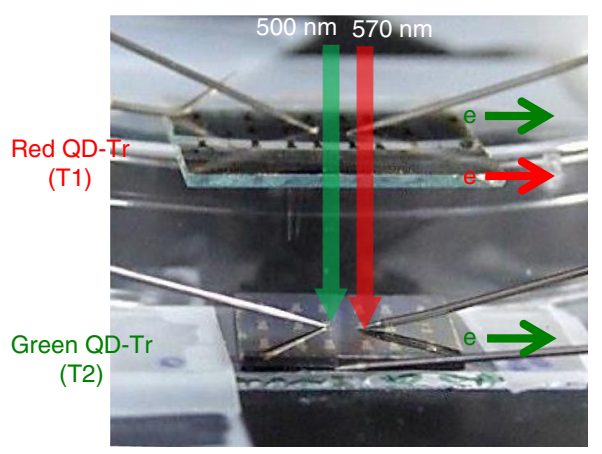

d

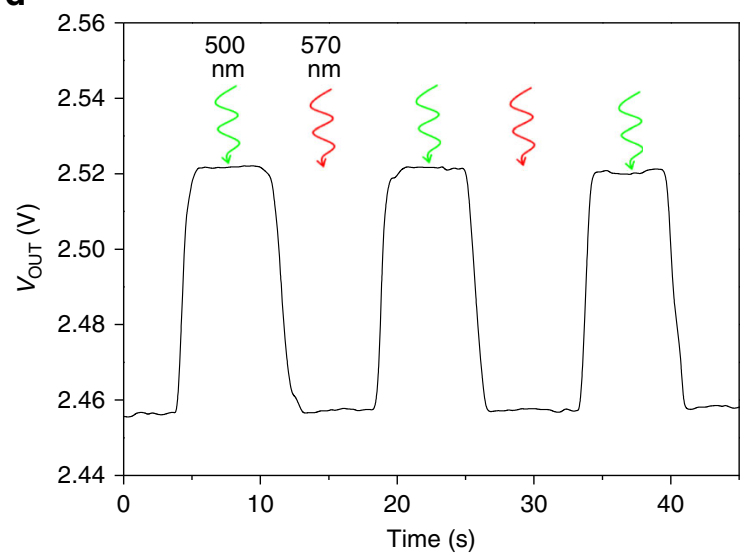

Fig. 5 Logic inverter using red- and green-quantum dot phototransistors. a Photoresponses of $1 \mathrm{RQ}-$ and $1 \mathrm{GQ}-\mathrm{OQO}$ phototransistors with respect to incident light wavelengths. Both transistors exhibit photoresponses at 500-nm wavelength illumination. For 570-nm-wavelength irradiation, the

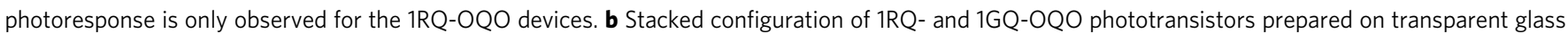
substrates for logic inverters. c Electro-optical NOT gate circuitry and resultant logic table. d Time-domain measurement of $V_{\text {out }}$ at alternated 500 - and 570-nm illumination wavelengths

may be explained by filling of the lowest-lying, longest-lived trap states that provide the highest photoconductive gain $\left(G_{\text {meas }}\right)$ at low light intensities, as shown by Konstantatos et al. ${ }^{5}$.

Note that $G_{\text {meas }}$ is one of the important figures of merit. This property is estimated from the ratio of the collected photocarrier rate at the electrodes and the incident photon flux, as given by $G_{\text {meas }}=R \cdot h v / e \eta$, where $R$ corresponds to the measured results shown in Fig. $4 \mathrm{~b}, h$ is the Planck constant, $v$ is the frequency of light, $e$ is the electron charge, and $\eta$ is the quantum efficiency. Figure $4 \mathrm{c}$ indicates that the $G_{\text {meas }}$ for 1 RQ-OQO phototransistors is in the $10^{2}-10^{4}$ range at $V_{\mathrm{G}}=60 \mathrm{~V}$, with $487-\mathrm{nm}$ illumination. However, we must also consider an inherent photoconductive gain $\left(G_{\text {inher }}\right)$, which includes the net absorption in the OQO channels, by eliminating the reflection $\left(R_{\text {light }}\right)$ and transmission $\left(T_{\text {light }}\right)$ of the devices ${ }^{31}$. Thus, $G_{\text {inher }}=G_{\text {meas }} /\left(1-R_{\text {light }}-T_{\text {light }}\right)$, because the majority of the photon flux is transmitted by the monolayered-QD configuration. Here, we obtained a $G_{\text {inher }}$ of $10^{5}-10^{7}$ for a light power of $5 \mu \mathrm{W}-5 \mathrm{nW}$ (see Supplementary Note 4). This implies that the photocarrier generation is highly efficient, even for a tiny amount of incident photons. We further investigated and compared the photoconductive gains using the photoresponse decay time $\left(\tau_{\text {lifetime }}\right)$ and the carrier transit time $\left(\tau_{\text {transit }}\right)$ of the OQO device, where $G_{\tau}=\tau_{\text {lifetime }} / \tau_{\text {transit }}$. The measured decay time can be represented by two components with fast and slow decays using a two-exponential fitting method, as shown in Supplementary Fig. 11 (see also Supplementary Note 5).
Furthermore, $\tau_{\text {transit }}=L^{2} / \mu \cdot V_{\mathrm{SD}}$, where $L$ is the length between the source and drain electrodes, $\mu$ is the channel mobility, and $V_{\mathrm{SD}}$ is the voltage difference between the electrodes. Here, $\tau_{\text {transit }}=5.0 \times 10^{-7} \mathrm{~s}$, obtained using $L=50 \mu \mathrm{m}, \mu=10 \mathrm{~cm}^{2} \mathrm{~V}^{-1} \mathrm{~s}^{-1}$, and $V_{\mathrm{SD}}=5 \mathrm{~V}$. Our prediction for $G_{\tau}$ is located in the $1.2 \times 10^{4}-2.2 \times 10^{5}$ range, as indicated by the empty and filled triangles in Fig. $4 c$, for an irradiation power of $5 \mu \mathrm{W}$, for example. Therefore, this estimation of the upper and lower bounds of $G_{\tau}$ using the fast and slow decay times indicates that the use of $G_{\text {inher, }}$, considering the net absorption, is both practical and accurate for our OQO configuration, because $G_{\text {inher }}$ is situated midway between the upper and lower bounds of $G_{\tau}$.

Based on these results, we prepared three different OQO phototransistors with monolayered QDs for R, G, or B colors. We chose 403- (near UV) and 487-nm (sky blue) illumination wavelengths as sample cases, to investigate the color-selective detection of the three devices. Figure $4 \mathrm{~d}$ shows that the $403-\mathrm{nm}$ irradiation on the 1RQ- and 1GQ-OQO phototransistors yields higher $R$ than the $487-\mathrm{nm}$ illumination; this is because of the larger absorption, as discussed with regard to Fig. $2 \mathrm{~d}$. The $R$ of the $1 \mathrm{GQ}-\mathrm{OQO}$ phototransistor reaches $14,900 \mathrm{AW}^{-1}$ at a $V_{\mathrm{G}}$ of $60 \mathrm{~V}$. The response of the $1 \mathrm{BQ}-\mathrm{OQO}$ device is notable in that it is activated at $403-\mathrm{nm}$ wavelength, but there is almost no response for the 487-nm illumination; this is because of the lack of absorption. This result suggests highly sensitive RGB-color- 

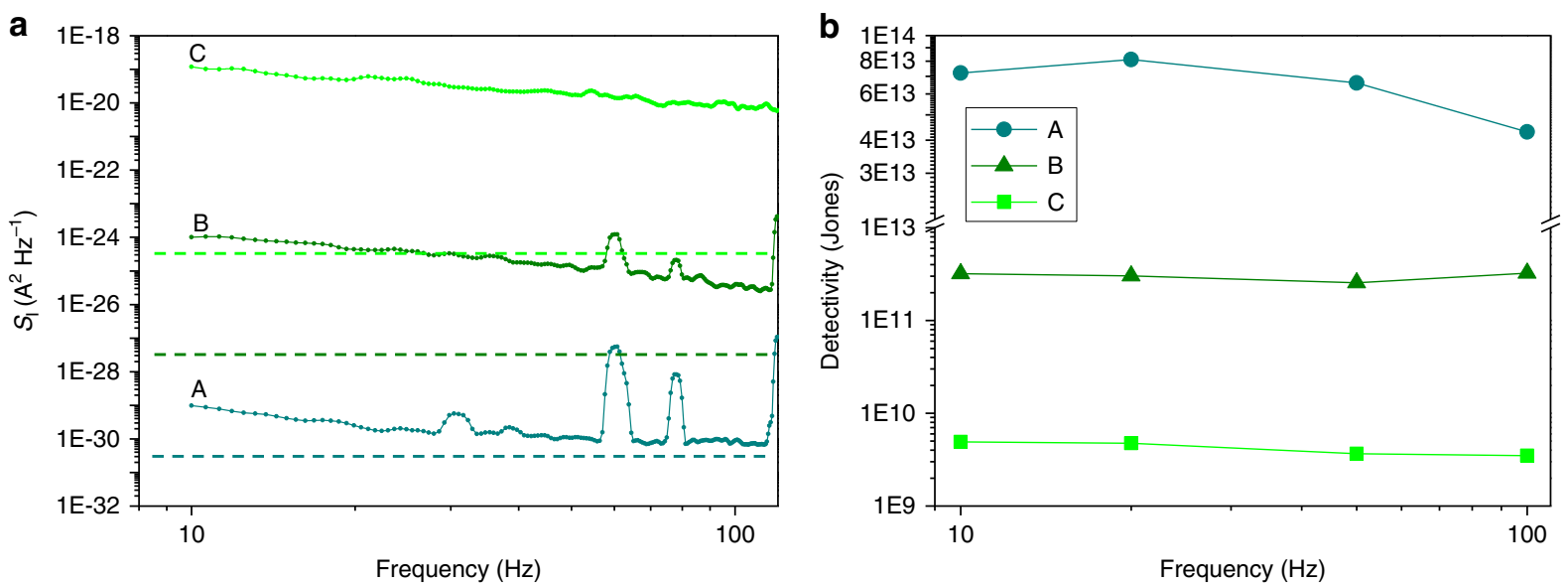

Fig. 6 Noise power spectral density and specific detectivity. a $S_{I}$ as function of modulation frequency in 1GQ-OQO phototransistor. A, B, and C denote three different measurement regimes, corresponding to $V_{G}$ values of $-18,-15$, and $12 \mathrm{~V}$, respectively, (see also, Supplementary Fig. 13a). b Estimation of $D^{\star}$ for three regimes of operation vs. modulation frequency

selective detection when the $R$ threshold levels are chosen appropriately at the desired wavelength of illumination.

Logic inverter using color-selective detection. Figure 5 shows the logic circuit of an electro-optical NOT gate (inverter) as a simple embodiment of color-selective detection. Taking into consideration the flexibility in the control of parameters such as the QD absorption energy band, the illumination wavelength with power, and $V_{\mathrm{G}}$, we employed 1RQ- and 1GQ-OQO phototransistors for color-selective detection. Each transfer curve was measured at two different illumination wavelengths, 500 and 570 $\mathrm{nm}$, as depicted in Fig. 5a. From these curves, an operating condition for a NOT gate that outputs a voltage representing the opposite logic level to its input can be chosen. We selected the gate voltages $\left(V_{\mathrm{G} 1}=10 \mathrm{~V}\right.$ and $\left.V_{\mathrm{G} 2}=12 \mathrm{~V}\right)$ indicated by the colored circles and filled dots. Figure 5b shows a stacked configuration of 1RQ- and 1GQ-OQO phototransistors (T1 and T2, respectively), which has the advantage of transparent OQO channels (see Supplementary Note 6) for light illumination. When devices are illuminated with light of 500- or 570-nm wavelength, the read-out voltage $\left(V_{\text {OUT }}\right)$ outputs high or low levels between the source and drain electrodes of each device, respectively. At $500-\mathrm{nm}$ irradiation, the voltage drop across $\mathrm{T} 1$ is larger than that across $\mathrm{T} 2$, because of the higher impedance of $\mathrm{T} 1$, as shown in Fig. $5 \mathrm{a}$. On the contrary, $V_{\text {OUT }}$ at $570 \mathrm{~nm}$ becomes low because of the small impedance of T1, which is due to the large photo-induced charges in the $\mathrm{T} 1$ channel. These results are summarized in the table included in Fig. $5 \mathrm{c}$ and correspond to a standard NOT gate. Therefore, we successfully demonstrated the on-and-off switching characteristics of $V_{\text {OUT }}$ using two different illumination wavelengths in the time domain, as shown in Fig. $5 \mathrm{~d}$. We may further improve the response time using fast-switching optical sources, because here we employed optical parametric oscillator (OPO) laser system (Opolette HR 355, OPOTEK) requiring $2.3 \sim 2.4 \mathrm{~s}$ for switching the wavelengths in high accuracy. This kind of logic circuits for color-selective detection provides opportunities for color-filterless photodetection. CMOS image sensors, for example, have a critical problem of light crossover between adjacent subpixels, due to the presence of color filters. However, our color-filterless, stacked configuration may solve this problem, through the use of highly transparent monolayered-QD OQO phototransistors. These devices can also be applied to high-resolution image sensors without lateral subpixels for colors, which is made possible by the realization of this vertically stacked RGB-tandem structure.
Noise and detectivity. On the other hand, noise is another important figure of merit that must be carefully characterized. Therefore, we measured the noise power spectral density $\left(S_{\mathrm{I}}\right)$ and $D^{*}$, as shown in Fig. 6, using an SR570 low-noise current amplifier and a HP89441 vector signal analyzer. The OQO phototransistors for noise measurement and $D^{*}$ were designed to have a square shape $\left(50 \times 50 \mu \mathrm{m}^{2}\right)$. Among the various kinds of noise, shot noise, Johnson noise, and $1 / f$ noise are dominant in phototransistors $5,10,32,33$. The shot noise limit is expressed as $\left.<I_{\mathrm{SN}}^{2}\right\rangle=2 q I_{\mathrm{D}} \Delta f$ and the Johnson noise is $4 k T \Delta f / R_{\mathrm{D}}$, where $q$ is the electron charge, $I_{\mathrm{D}}$ is the dark current, $\Delta f$ is the noise bandwidth, $k$ is the Boltzmann constant, $T$ is temperature, and $R_{\mathrm{D}}$ is the detector resistance under dark conditions. We chose three different $V_{\mathrm{G}}$ values representing the subthreshold (A), ohmic (B), and near-saturation (C) regimes (see Supplementary Fig. 13a).

In Fig. 6a, the flicker $1 / f$ noise, $S_{I} \sim 1 / f$ increases with respect to increasing drain current levels, as indicated by $\mathrm{A}, \mathrm{B}$, and $\mathrm{C}$ for the 1GQ-OQO phototransistors. The shot noise limit at each current level is also plotted in dotted lines. We do not consider the Johnson noise, because this component is one order of magnitude lower than the shot noise in our devices (see Supplementary Note 7). In the subthreshold regime (A), the measured $S_{\mathrm{I}}$ is closer to the shot noise limit at high frequencies of modulation than in other operating regimes. This implies that we can achieve extremely low noise levels by controlling $V_{\mathrm{G}}$, which is a unique advantage of phototransistors. Based on this noise analysis, the photodetector sensitivity can be explicitly expressed using the $D^{*}$ parameter. $D^{*}$ can be rewritten as $D^{*}=\sqrt{A \Delta f} / \mathrm{NEP}=R \sqrt{A / S_{\mathrm{I}}}$, where $A$ is the effective area of the detector in $\mathrm{cm}^{2}$ and $R$ is in $\mathrm{AW}^{-1}$, measured under the same conditions as $S_{\mathrm{I}}$ (see more details in Methods and Supplementary Fig. 13b). Figure 6b represents the calculated results for $D^{*}$ vs. the modulation frequency with respect to $\mathrm{A}, \mathrm{B}$, and C. An inverse relationship between $D^{*}$ and $I_{D}$ is observed, as expected. The $D^{*}$ of the $1 \mathrm{GQ}-\mathrm{OQO}$ phototransistor reaches $8.1 \times 10^{13}$ Jones at $20-\mathrm{Hz}$ frequency in regime A. Such ultrasensitive photodetection can be attributed to the proposed OQO configuration with monolayered QDs, which facilitates effective photocarrier control. This $D^{*}$ is approximately $17 \%$ higher than the previous record for QD-graphene hybrid phototransistors $\left(7 \times 10^{13} \text { Jones }\right)^{11}$. Moreover, $D^{*}$ can be further enhanced by reducing the flicker $1 / f$ noise, when the surface defects of QDs are controlled to reduce trap sites or defects in the channel. ${ }^{10}$ 


\section{Discussion}

We demonstrated ultrasensitive photodetection using monolayered QDs buried in amorphous-oxide SIZO phototransistors. The effectively large interfacial areas of the QDs buried in the OQO configuration facilitates the observation of fast charge separation and charge transport caused by the high-mobility SIZO layers. Hence, we achieved RGB-color detection exhibiting a $G_{\text {meas }}$ value as high as $10^{7}$, which is obtained via roomtemperature deposition of the SIZO layers. By combining the transparent and color-sensitive characteristics, a logic circuit for color-selective detection was successfully demonstrated using vertically stacked multi-color OQO phototransistors without color filters. The concept proposed in this study also allows monolithic integration with a CMOS-compatible process, which presents a basis for myriad emerging applications in color detection with sensitivity, transparency, and even flexibility.

\section{Methods}

Device fabrication. We synthesized three different species of red (CdSe), green (CdSeS), and blue (CdS) QDs, of approximately $4.5 \mathrm{~nm}$ in diameter. More details of the QD synthesis and purification are presented in the Supplementary Method 1. Amorphous SIZO TFTs with a bottom gate and top electrodes were fabricated using conventional photolithography. The bottom amorphous SIZO film was deposited on a $\mathrm{SiO}_{2}(200 \mathrm{~nm}) / \mathrm{Si}$ substrate using room-temperature RF sputtering at $0.56-\mathrm{Pa}$ pressure in mixed $\mathrm{Ar} / \mathrm{O}_{2}$ gases. After spin-coating of the QDs, the top amorphous SIZO film was deposited under the same conditions as for the bottom SIZO film deposition. Then, a low-temperature, annealing process was conducted at $150{ }^{\circ} \mathrm{C}$. The bottom and top SIZO layers had thicknesses of 40 and $30 \mathrm{~nm}$, respectively. The channel widths and lengths were 250 and $50 \mu \mathrm{m}$, respectively, expect for OQO phototransistors for noise and detectivity measurement. Regarding the thickness of QD layers, we controlled the concentration of QD solution and the speed of spin coating. For example, $1 \mathrm{wt} \%$ QD in cyclohexane solution with a coating speed at $2000 \mathrm{rpm}$ was applied for six-layered QD films.

Photodetection measurement. The photodetection measurement was conducted using a custom-built confocal scanning microscope. All the measurements were performed using monochromatic solid-state CW diode lasers of 487- and 403-nm wavelength except for spectral R (responsivity) measurement as shown in Fig. 2c. For such spectral R measurements (Fig. 2c) together with logic-inverter measurement (Fig. 5), an optical parametric generator-amplifier (OPG-OPA) pulse laser (Opolett HR355, OPOTEK) was used as a tunable light source $(400-700 \mathrm{~nm})$. The spatial photocurrent images in Fig. $1 \mathrm{~b}$ were obtained using laser beam scanning through an objective lens ( $\times 50,0.8$ numerical aperture). The objective lens of the microscope focused the beam to a diffraction-limited spot of $500 \mathrm{~nm}$ in diameter on the device. The source-drain voltage is $5 \mathrm{~V}$ for all the measurement, i.e., transfer curves, noise measurement, etc.

Detectivity measurement. The detectivity is related to the NEP by following expression: $D^{*}=\sqrt{A \Delta f} / \mathrm{NEP}$, where $\mathrm{A}$ is the photosensitive area of the device and $\Delta f$ is the detection bandwidth. The NEP is defined as the amount of incident light power inducing a photocurrent equal to the noise current, which is given by: $\mathrm{NEP}=\sqrt{\left\langle I_{\mathrm{n}}^{2}\right\rangle} / R=\sqrt{S_{\mathrm{I}} \Delta f} / R$, where $\left\langle I_{\mathrm{n}}^{2}\right\rangle$ is the time-averaged square of the total noise current and $R$ is the responsivity. Here, we measured the noise spectral density $\left(S_{\mathrm{I}}\right)$ of the drain current under dark conditions using an SR570 low-noise current amplifier and a Hewlett Packard 89441 vector signal analyzer. The measurement was conducted in an electrically and optically shielded probe station. The OQO phototransistors for noise measurement were designed to have a square shape $\left(50 \times 50 \mu \mathrm{m}^{2}\right)$ photosensitive area $(A)$.

Data availability. The data that support the findings of this study are available from the corresponding authors on request.

Received: 9 September 2016 Accepted: 3 August 2017

Published online: 10 October 2017

\section{References}

1. Konstantatos, G. \& Sargent, E. H. Nanostructured materials for photon detection. Nat. Nanotech. 5, 391-400 (2010).

2. Saran, R. \& Curry, R. J. Lead sulphide nanocrystal photodetector technologies. Nat. Photon. 10, 81-92 (2016).
3. Guo, F. et al. A nanocomposite ultraviolet photodetector based on interfacial trap-controlled charge injection. Nat. Nanotech. 7, 798-802 (2012).

4. Liu, K., Sakurai, M. \& Aono, M. ZnO-based ultraviolet photodetectors. Sensors 10, 8604-8634 (2010).

5. Konstantatos, G., Clifford, J., Levina, L. \& Sargent, E. G. Sensitive solution-processed visible-wavelength photodetectors. Nat. Photon. 1, 531-534 (2007).

6. Lee, J. S., Kovalenko, M. V., Huang, J., Chung, D. S. \& Talapin, D. V. Band-like transport, high electron mobility and high photoconductivity in all-inorganic nanocrystal arrays. Nat. Nanotech. 6, 348-352 (2011).

7. Liu, X. et al. Photo-modulated thin film transistor based on dynamic charge transfer within quantum-dots-InGaZnO. Appl. Phys. Lett. 104, 113051 (2014).

8. Lee, S. M. et al. Enhanced photocurrent of Ge-doped $\mathrm{InGaO}$ thin film transistors with quantum dots. Appl. Phys. Lett. 106, 031112 (2015).

9. Shin, S. W., Lee, K.-H., Park, J.-S. \& Kang, S. J. Highly transparent, visible-light photodetector based on oxide semiconductors and quantum. ACS Appl. Mater. Interf. 7, 19666-19671 (2015).

10. Konstantatos, G. et al. Ultrasensitive solution-cast quantum dot photodetectors. Nature 442, 180-183 (2006).

11. Konstantatos, G. et al. Hybrid graphene-quantum dot phototransistors with ultrahigh gain. Nat. Nanotech. 7, 363-368 (2012).

12. Ginger, D. S. \& Greenham, N. C. Charge injection and transport in films of CdSe nanocrystals. J. Appl. Phys. 87, 1361-1368 (2000).

13. $\mathrm{Yu}, \mathrm{D}$. et al. Electronic transport of n-type CdSe quantum dot films: effect of film treatment. J. Appl. Phys. 99, 104315 (2006).

14. Hwang, D. K. et al. Ultrasensitive PbS quantum-dot-sensitized InGaZnO hybrid photoinverter for near-infrared detection and imaging with high photogain. NPG Asia Mater. 8, e233 (2016).

15. Kufer, D. et al. Hybrid 2D-0D MoS2 -PbS quantum dot photodetectors. $A d v$ Mater. 27, 176-180 (2015).

16. Kufer, D., Lasanta, T., Bernechea, M. \& Koppens, F. H. L. Interface engineering in hybrid quantum dot-2D phototransistors. ACS Photonics 3, 1324-1330 (2016).

17. Turyanska, L. et al. Ligand-induced control of photoconductive gain and doping in a hybrid graphene-quantum dot transistor thin-film transistors. $A d v$. Elec. Mater 1, 1500062 (2015).

18. Fortunato, E., Barquinha, P. \& Martins, R. Oxide semiconductor thin-film transistors: a review of recent advances. Adv. Mater. 24, 2945-2986 (2012).

19. Kamyya, T., Nomura, K. \& Hosono, H. Present status of amorphous In-Ga-Zn-O thin-film transistors. Sci. Technol. Adv. Mater. 11, 044305 (2010).

20. Chong, E., Chun, Y. S. \& Lee, S. Y. Amorphous silicon-indium-zinc oxide semiconductor thin film transistors processed below $150{ }^{\circ} \mathrm{C}$. Appl. Phys. Lett. 97, 102102 (2010).

21. Chong, E., Kim, S. H. \& Lee, S. Y. Role of silicon in silicon-indium-zinc-oxide thin-film transistor. Appl. Phys. Lett. 97, 252112 (2010).

22. Bae, H. S., Yoon, M. H., Kim, J. H. \& Im, S. Photodetecting properties of ZnO-based thin-film. Appl. Phys. Lett. 83, 5313-5315 (2003).

23. Kim, T.-H. et al. Full-colour quantum dot displays fabricated by transfer printing. Nat. Photon 5, 176-182 (2011).

24. Choi, J. Y., Heo, K., Cho, K.-S., Hwang, S. W., Kim, S. \& Lee, S. Y. Engineering of band gap states of amorphous $\mathrm{SiZnSnO}$ semiconductor as a function of $\mathrm{Si}$ doping concentration. Sci. Rep. 6, 36504 (2016).

25. Bao, J. \& Bawendi, M. G. A colloidal quantum dot spectrometer. Nature $\mathbf{5 2 3}$ 67-70 (2015)

26. Talapin, D. V. \& Murray, C. B. PbSe nanocrystal solids for $n$ - and p-channel thin film field-effect transistors. Science 310, 86-89 (2005).

27. Geyer, S. et al. Charge transport in mixed CdSe and CdTe colloidal nanocrystal films. Phys. Rev. B 82, 155201 (2010).

28. Morgan, N. Y. et al. Electronic transport in films of colloidal CdSe nanocrystals. Phys. Rev. B 66, 075339 (2002).

29. Jarosz, M. V., Porter, V. J., Fisher, B. R., Kastner, M. A. \& Bawendi, M. G. Photoconductivity studies of treated CdSe quantum dot films exhibiting increased exciton ionization efficiency. Phys. Rev. B 70, 195327 (2004).

30. Beard, M. C., Turner, G. M. \& Schmuttenmaer, C. A. Size-dependent photoconductivity in CdSe nanoparticles as measured by time-resolved Terahertz spectroscopy. Nano Lett. 2, 983-987 (2002).

31. Hernandez-Sosa, G., Coates, N. E., Valouch, S. \& Moses, D. High photoconductive responsivity in solution-processed polycrystalline organic composite films. Adv. Func. Mater. 21, 927-931 (2011).

32. Park, J. C. et al. Low-frequency noise in amorphous indium-gallium-zinc oxide thin-film transistors from subthreshold to saturation. Appl. Phys. Lett. 97 122104 (2010).

33. Balandin, A. A. Low-frequency $1 /$ f noise in graphene devices. Nat. Nanotech. 8 , 549-555 (2013).

\section{Acknowledgements}

This work was supported by a National Research Foundation grant, funded by the Korean Government (MEST, No. 2012-0001214). We thank J. S. Lee, C.-Y. Jeong and 
H.I. Kwon for noise measurements and analysis, T.H. Shin for time-resolved photoluminescence measurements, J. Lee for TEM analysis, and S. M. Han for SIZO deposition, J.E. Jin, J.-H. Park, and C.-W. Lee for helpful discussions.

\section{Author contributions}

K.-S.C., K.H., J.Y.C., S.H., and S.Y.L. designed the experiments. K.-S.C., K.H., C.-W.B., J.Y.C, and H.J. performed the experiments and analyses. S.H. and S.Y.L advised and supervised the project. K.-S.C., K.H., C.-W.B, and H.J. wrote the paper.

\section{Additional information}

Supplementary Information accompanies this paper at doi:10.1038/s41467-017-00893-X.

Competing interests: The authors declare no competing financial interests.

Reprints and permission information is available online at http://npg.nature.com/ reprintsandpermissions/
Publisher's note: Springer Nature remains neutral with regard to jurisdictional claims in published maps and institutional affiliations.

(c) (i) Open Access This article is licensed under a Creative Commons Attribution 4.0 International License, which permits use, sharing, adaptation, distribution and reproduction in any medium or format, as long as you give appropriate credit to the original author(s) and the source, provide a link to the Creative Commons license, and indicate if changes were made. The images or other third party material in this article are included in the article's Creative Commons license, unless indicated otherwise in a credit line to the material. If material is not included in the article's Creative Commons license and your intended use is not permitted by statutory regulation or exceeds the permitted use, you will need to obtain permission directly from the copyright holder. To view a copy of this license, visit http://creativecommons.org/ licenses/by/4.0/.

(C) The Author(s) 2017 